\title{
ARTICULACIÓN INTERSECTORIAL Y COMPLEJOS PRODUCTIVOS. UN ANÁLISIS DEL SISTEMA PRODUCTIVO ANDALUZ
}

\author{
Isidoro ROMERO LUNA*
}

\section{RESUMEN}

En este trabajo se resalta, en el plano teórico, la importancia de los encadenamientos productivos en el desarrollo endógeno como fuente de externalidades estáticas y dinámicas. Desde esta perspectiva, se analiza la estructura productiva de la economía andaluza a partir de las tablas input-output regionales de 1995 (Instituto de Estadística de Andalucía, 1999), presentando una clasificación de las ramas productivas en función de su grado de integración en el sistema productivo regional. Así mismo, se identifican tres complejos productivos -agroalimentario, de la construcción y turístico-como núcleos centrales de la estructura productiva regional. Finalmente, se estudia la estructura interna de estos complejos y las interrelaciones que se establecen entre ellos.

\section{ABSTRACT}

In this article, from a theoretical perspective, we emphasize the importance of productive linkages in endogenous development, as a source of static and dynamic externalities. From this point of view, we analyse the andalusian productive structure using the regional input-output table for 1995 (Instituto de Estadística de Andalucía, 1999). In this respect, we classify the productive branches in Andalusia according to their level of integration into the regional production system. Furthermore, we identify three productive clusters -agro-food complex, construction, and tourismas the centres of the regional productive structure. Finally, we study the internal structure of these clusters and the interrelation among them.

\footnotetext{
* Dpto. Economía Aplicada I. Universidad de Sevilla
} 


\section{INTRODUCCIÓN}

Una manifestación significativa del atraso económico que mantiene Andalucía respecto a otras regiones españolas y europeas es la desarticulación de su tejido productivo, circunstancia ésta que diversos estudios han puesto de manifiesto ${ }^{1}$. En este sentido, probablemente el fenómeno más llamativo sea la presencia de "enclaves industriales", dependientes de iniciativas y capitales exógenos a la región, que se aprovisionan en el exterior de los inputs intermedios que necesitan y destinan su output fundamentalmente a la exportación, quedando desvinculados de la dinámica económica regional.

En muchos casos, las grandes empresas originarias de otras zonas no se integran en el sistema productivo regional y, al mantener sus centros de decisión en el exterior, incrementan la dependencia externa y la vulnerabilidad de la economía andaluza ante eventuales decisiones de deslocalización. Estas empresas multinacionales optan en ocasiones por trasladar sus plantas hacia regiones con costes laborales menores, generando graves perjuicios socioeconómicos en las áreas que abandonan. A este respecto, pueden señalarse casos como los de Gillete en Alcalá de Guadaíra o Suzuki en Linares.

Por todo ello, la política de desarrollo regional debe prestar especial atención a la movilización y la valorización de los recursos autóctonos (naturales, humanos, culturales e institucionales) a partir de la iniciativa empresarial regional o de grandes empresas externas que se integren realmente en el sistema productivo andaluz. Desde esta perspectiva resulta interesante identificar aquellos sectores cuyas cadenas de valor están realmente endogeneizadas en el sistema productivo andaluz, es decir, aquellas ramas productivas que están fuertemente vinculadas y enraizadas en el sistema productivo regional a través de encadenamientos productivos (hacia adelante y hacia atrás) con otras actividades.

En este trabajo, a partir de los datos que proporciona el Sistema de Cuentas Económicas de Andalucía. Marco Input-Output 1995 (Instituto de Estadística de Andalucía, 1999), se estudian las pautas de articulación productiva de la economía andaluza. Para ello se comienza con unas consideraciones preliminares relativas al papel de los encadenamientos productivos intersectoriales en el desarrollo endógeno. A continuación, se presenta una clasificación de los sectores productivos andaluces en función de la intensidad de sus encadenamientos productivos directos con otras ramas de actividad. De este modo, resulta posible identificar tres complejos productivos -el agroalimentario, el de la construcción y el turístico-que conforman el núcleo esencial del tejido productivo endógeno de la economía andaluza. Seguidamente, se

1. Véase Aurioles Martín (1989), Delgado Cabeza (1981 y 1995), Moral Pajares (1998) y, desde una óptica empresarial, Guzmán Cuevas y Liñán Alcalde (1997), entre otros ejemplos. 
analizan la estructura interna de estos complejos y las interrelaciones que se establecen entre ellos.

\section{RELACIONES INTERSECTORIALES, ECONOMÍAS EXTERNAS Y DESARROLLO ENDÓGENO}

El estudio de los vínculos intersectoriales se asocia habitualmente al análisis de los efectos de arrastre o de empuje de unas ramas productivas respecto a otras. En presencia de un eslabonamiento productivo hacia atrás, el crecimiento de la producción de una industria estimula la actividad de aquellas otras que le proporcionan inputs intermedios; mientras que, en el caso de un eslabonamiento hacia adelante, el crecimiento de la producción de una industria proveedora de un bien intermedio puede impulsar la actividad en aquellas otras que lo utilizan en sus procesos de transformación, al beneficiarse de un mejor aprovisionamiento o de precios menores. No obstante, los encadenamientos productivos entre ramas de actividad son el origen de otras externalidades de diversa naturaleza, tanto de carácter estático como dinámico.

Las externalidades estáticas explican las decisiones de localización de las empresas en función del incentivo que supone para éstas la reducción de los costes o la elevación de productividad derivadas de la explotación del efecto externo; sin embargo, este tipo de externalidades no puede explicar el desarrollo de un proceso sostenido de elevación de la productividad y, por lo tanto, de crecimiento industrial. Los efectos de una economía externa de carácter estático son simultáneos a la propia existencia de la externalidad y se agotan inmediatamente a su aprovechamiento ${ }^{2}$.

En este sentido, A. O. Hirschman analizó el papel estratégico que pueden jugar en el desarrollo de las economías atrasadas los sectores que presentan eslabonamientos productivos más intensos ${ }^{3}$. Hirschman observó que los encadenamientos intersectoriales hacia atrás y hacia adelante podían actuar como mecanismos de inducción al desarrollo y defendió que la política económica debía tenerlos en cuenta y activarlos para aprovechar sus efectos. Junto a los encadenamientos desarrollados, observables a partir de los flujos de consumos intermedios entre sectores, existen encadenamientos potenciales, que se mantienen en estado latente en tanto no han dado lugar a un estímulo lo suficientemente fuerte como para suscitar decisiones localización de nuevas industrias. Realmente, son estos encadenamientos potenciales los que resultan más interesantes para la política de desarrollo, puesto que muestran una vía de crecimiento para la economía que se esté considerando. Los encadenamientos potenciales

2. Glaeser Edward L., D. Kallal, Hedi; Scheinkman, José A. y Shleifer, Andrei (1992); "Growth in Cities", Journal of Political Economy, vol. 100, nº 6, p. 1148.

3. Hirschman, Albert O. (1961): La estrategia del desarrollo económico. Fondo de Cultura Económica. México D. F. 
albergan economías externas de localización potenciales, que estimulan el establecimiento de nuevas empresas. Concretamente, los encadenamientos hacia atrás impulsan la aparición de empresas cuya producción es utilizada como insumo por una rama productiva previamente establecida, que representa un mercado potencial atractivo. Por su parte, los encadenamientos hacia adelante estimulan la aparición de empresas que emplean la producción de la rama suministradora establecida como insumo en sus procesos productivos y que, por lo tanto, se beneficiarán de las facilidades para aprovisionarse de los inputs básicos que necesitan.

Por otro lado, los encadenamientos intersectoriales generan también externalidades dinámicas que actúan impulsando el crecimiento sostenido de la productividad de las actividades económicas. Entre ellas las que han merecido más atención en la literatura son los efectos de difusión tecnológica (technological spillovers), es decir, los incrementos de productividad derivados de los flujos de información entre los agentes económicos que propician la difusión de las innovaciones ${ }^{4}$, a través de múltiples vías como la imitación, el espionaje industrial, la movilidad de la mano de obra especializada entre empresas, etc.; De esta forma, las innovaciones o mejoras originadas en una empresa pueden elevar la productividad de otras sin compensación por parte de éstas últimas. La posibilidad de aprender de otros representa, en este sentido, un impulso potente para el avance de la productividad.

Pueden distinguirse dos tipos de technological spillovers: los rent-spillovers y los technological spillovers puros ${ }^{5}$. Los primeros están relacionados con el flujo de bienes entre las empresas. La innovación y el desarrollo tecnológico incrementan la calidad de los productos, pero esta mejora no suele reflejarse plenamente en el precio debido a las presiones competitivas y a la elasticidad de la demanda. De esta forma, innovaciones en una empresa que fabrica un input demandado por otra elevan la productividad de la segunda, en lo que constituye un efecto derrame de la propia innovación tecnológica. Esta vía de transmisión de los beneficios de la innovación afecta de manera especial a las ramas de actividad que están ligadas por eslabonamientos productivos.

Por su parte, las externalidades tecnológicas puras no están relacionadas con ningún flujo material de productos, sino con flujos intangibles de información y conocimiento. Por lo tanto, se trata de externalidades no pecuniarias, en tanto no se materializan a través de transacciones en el mercado, por lo que resultan más etéreas y de difícil medición. En cualquier caso, cabe esperar que las industrias que estén relacionadas en virtud de algún eslabonamiento productivo resulten un canal más frecuente para la manifestación de estos efectos. De hecho, la literatura sobre esta cuestión utiliza

4. Glaeser et alia; (1992): op. cit., pp. 1126-1152.

5. Verspagen, B. y De Loo, I. (1998): “Technology Spillovers Between Sectors and Over Time”, Maastricht Economic Research Institute on Innovation and Technology (MERIT), Working Paper for the 12th International Conference on Input-Output Techniques, p. 2. 
para cuantificar los efectos de derrame "matrices de flujos tecnológicos" que, en una de las aproximaciones alternativas desarrolladas, se construyen a partir de las tablas input-output.

En las últimas décadas han cobrado mucha importancia las teorías del desarrollo endógeno que defienden una estrategia de desarrollo orientada de abajo-arriba y fundamentada en la valorización de los recursos autóctonos de un territorio. Desde esta perspectiva, adquiere un renovado interés la noción de articulación productiva como manifestación de fortaleza del tejido productivo de una región. Una mayor vertebración del sistema productivo permite el aprovechamiento de las externalidades estáticas y dinámicas que subyacen a los encadenamientos productivos, impulsando el crecimiento de la productividad. Por lo tanto, es conveniente mantener un sistema productivo diverso y articulado, que combine una cierta cohesión interna con una sólida inserción externa en una economía mundial globalizada.

\section{UNA CLASIFICACIÓN DE LAS RAMAS PRODUCTIVAS EN FUNCIÓN DE SU INTEGRACIÓN EN EL SISTEMA PRODUCTIVO ANDALUZ}

La intensidad de los encadenamientos directos ${ }^{6}$ hacia adelante y hacia atrás de un sector respecto a otro puede medirse, partiendo de la información que proporcionan las tablas input-output, a través de unos índices simples propuestos originalmente por Chenery y Watanabe en 1958. En concreto, la capacidad de arrastre de un sector se puede cuantificar a partir de la suma por columnas de los coeficientes técnicos de producción como sigue ${ }^{7}$ :

$$
\mu_{j}=\sum_{i=1}^{n} \frac{x_{i j}}{X_{j}}=\sum_{i=1}^{n} a_{i j} \quad \text { para } \mathrm{j}=1, \ldots, \mathrm{n}
$$

Este índice representa las necesidades que cada rama tiene de los inputs intermedios que le proporcionan el resto de ramas productivas y puede interpretarse como la proporción, en tantos por uno, del valor de la producción de un sector que procede de los consumos intermedios de otras ramas de actividad.

6. Los encadenamientos directos no recogen las necesidades indirectas que se generan cuando, en respuesta a un estímulo inicial de la demanda, el conjunto de las ramas productivas incrementan su output y obligan al sistema productivo a aumentar su producción de bienes intermedios. Para cuantificar los efectos totales se recurre a los elementos de la matriz inversa de Leontief. No obstante, en función de los objetivos de este trabajo, consideramos más adecuado realizar el análisis a partir de los eslabonamientos directos.

7. $x_{i j}$ representa los consumos intermedios del sector $j$ de bienes elaborados por el sector productivo $i$ y $X_{j}$ la producción total de cada sector. 
Por otra parte, la capacidad de empuje de una actividad productiva puede medirse a través del índice siguiente:

$$
\omega_{i}=\sum_{j=1}^{n} \frac{x_{i j}}{X_{i}}=\sum_{j=1}^{n} d_{i j} \quad \text { para } \mathrm{i}=1, \ldots, \mathrm{n} ;
$$

Este indicador no es más que la suma por filas de los coeficientes de distribución y representa el peso de las ventas intermedias en el conjunto de la producción de una rama de actividad, o bien la parte de la producción de una rama de actividad que no se destina a la demanda final, sino a las necesidades intermedias de otras ramas. Por lo tanto, este índice informa sobre la situación de un sector en la cadena del valor, indicando si tiene un carácter predominantemente final o intermedio.

Aplicando ambos indicadores a las últimas tablas input-output andaluzas disponibles, las estimadas por el Instituto de Estadística de Andalucía (1999) para el año 1995, se han calculado los eslabonamientos a partir de los consumos intermedios regionales, descontando, por lo tanto, el empleo que hacen las diversas ramas productivas de inputs procedentes del exterior de la comunidad autónoma andaluza. De esta forma, se ponen de manifiesto sólo las relaciones entre las unidades productivas establecidas en el territorio andaluz.

La consideración conjunta de estos dos coeficientes permite clasificar las ramas de actividad que conforman la economía regional en cuatro tipos, según presenten encadenamientos hacia atrás y hacia adelante superiores o inferiores a la media de las ramas productivas regionales, como puede observarse en el siguiente cuadro.

\section{CUADRO 1.}

\section{ClaSIFICACIÓN DE LAS RAMAS DE ACTIVIDAD SEGÚN} SUS ENCADENAMIENTOS DIRECTOS.

\begin{tabular}{|c|c|c|c|}
\cline { 3 - 3 } \multicolumn{2}{c|}{} & \multicolumn{2}{c|}{ Encadenamientos hacia adelante } \\
\cline { 3 - 4 } \multicolumn{2}{c|}{} & Superior a la media & Inferior a la media \\
\hline \multirow{2}{*}{$\begin{array}{c}\text { Encadenamientos } \\
\text { hacia atrás }\end{array}$} & Superior a la media & TIPO I & TIPO II \\
\cline { 2 - 4 } & Inferior a la media & TIPO III & TIPO IV \\
\hline
\end{tabular}

Dentro del primer grupo se encuadran las ramas de actividad que demandan inputs intermedios regionales y producen bienes intermedios requeridos por el resto del sistema productivo andaluz, lo que se refleja en unos encadenamientos directos hacia adelante y hacia atrás superiores a la media de las ramas productivas de la región. Se trata, por lo tanto, de los sectores que se encuentran más integrados en el tejido productivo regional. 


\section{CUADRO 2. \\ RAMAS PRODUCTIVAS TIPO I.} (Encadenamientos directos hacia atrás y hacia adelante superiores a la media)

\begin{tabular}{|c|l|c|c|}
\hline Código & \multicolumn{1}{|c|}{ Denominación } & $\begin{array}{c}\text { Encadenam. } \\
\text { hacia atrás }\end{array}$ & $\begin{array}{c}\text { Encadenam. } \\
\text { hacia adelante }\end{array}$ \\
\hline 7 & Extracción de carbones & 0,564 & 0,966 \\
\hline 11 & Extracción de minerales no metálicos ni energéticos & 0,402 & 0,722 \\
\hline 24 & Industria de la madera y del corcho (excepto muebles) & 0,323 & 0,589 \\
\hline 26 & $\begin{array}{l}\text { Edición, artes gráficas y reproducción de soportes } \\
\text { grabados }\end{array}$ & 0,368 & 0,506 \\
\hline 31 & Fabricación de cemento, cal, yeso y sus derivados & 0,407 & 0,399 \\
\hline 33 & Industrias del vidrio y de la piedra & 0,485 & 0,870 \\
\hline 46 & Reciclaje & 0,370 & 0,720 \\
\hline 47 & Producción y distribución de energía eléctrica & 0,361 & 0,873 \\
\hline 49 & Captación, depuración y distribución de agua & 0,303 & 0,824 \\
\hline 59 & Otros tipos de transporte terrestre y por tubería & 0,345 & 0,741 \\
\hline 60 & Transporte marítimo y fluvial & 0,351 & 0,584 \\
\hline 62 & Actividades anexas a los transportes & 0,837 & 0,442 \\
\hline 66 & Actividades auxiliares a la intermediación financiera & 0,412 & 0,525 \\
\hline 71 & Actividades jurídicas, de contabilidad, etc. & 0,913 & 0,921 \\
\hline 85 & Actividades asociativas & 0,320 & 0,990 \\
\hline 86 & Producciones de cine, vídeo, radio y televisión & 0,358 & 0,816 \\
\hline & Promedio de las ramas de actividad & $\mathbf{0 , 2 9 5}$ & $\mathbf{0 , 3 9 7}$ \\
\hline
\end{tabular}

Fuente: Elaboración propia a partir de datos extraídos del Sistema de Cuentas Económicas de Andalucía. Marco Input-Output 1995 (IEA; 1999).

Pertenecen a este grupo 16 ramas productivas. Figuran en él algunas actividades básicas - extractivas o energéticas-, como la extracción de carbones, la extracción de minerales no metálicos ni energéticos, la producción y distribución de energía eléctrica o la captación, depuración y distribución de agua. Del mismo modo, aparecen algunas industrias relacionadas con el sector de la construcción, como la industria de la madera y del corcho (excepto muebles), la fabricación de cemento, cal, yeso y sus derivados o las industrias del vidrio y de la piedra. Por último, se encuentran en este grupo las actividades de transporte. 
En el segundo grupo, formado por 17 ramas, se sitúan las actividades caracterizadas por una elevada capacidad de arrastre y por su orientación a la demanda final. Dentro de este grupo se encuentran fundamentalmente las industrias agroalimentarias, alguna otra rama industrial, como la fabricación de muebles o la construcción naval; el sector de la construcción y determinados servicios relacionados con la actividad turística, como los servicios de restaurantes y otros establecimientos para comer y beber o las actividades recreativas y culturales.

\section{CUADRO 3.}

RAMAS PRODUCTIVAS TIPO II.

(Encadenamientos directos hacia atrás superiores a la media y hacia adelante inferiores a la media)

\begin{tabular}{|c|l|c|c|}
\hline Código & \multicolumn{1}{|c|}{ Denominación } & $\begin{array}{c}\text { Encadenam. } \\
\text { hacia atrás }\end{array}$ & $\begin{array}{c}\text { Encadenam. } \\
\text { hacia adelante }\end{array}$ \\
\hline 10 & Extracción de minerales metálicos & 0,313 & 0,059 \\
\hline 12 & Industria cárnica & 0,464 & 0,231 \\
\hline 13 & Elaboración de conservas de pescado y de vegetales & 0,620 & 0,129 \\
\hline 14 & Fabricación de grasas y aceites & 0,702 & 0,167 \\
\hline 15 & Industrias lácteas & 0,463 & 0,079 \\
\hline 16 & Industrias de molinería, pan, galletas y pastelería & 0,379 & 0,169 \\
\hline 18 & Elaboración de vinos y alcoholes & 0,509 & 0,278 \\
\hline 19 & Elaboración de cerveza y bebidas no alcohólicas & 0,299 & 0,375 \\
\hline 42 & Construcción y reparación naval & 0,355 & 0,226 \\
\hline 44 & Fabricación de muebles & 0,336 & 0,084 \\
\hline 50 & Construcción de inmuebles y obras de ingeniería civil & 0,439 & 0,153 \\
\hline 57 & $\begin{array}{l}\text { Restaurantes y otros establecimientos para comer } \\
\text { yeber }\end{array}$ & 0,371 & 0,029 \\
\hline 65 & Seguros y planes de pensiones & 0,533 & 0,339 \\
\hline 72 & $\begin{array}{l}\text { Servicios técnicos de arquitectura e ingeniería, } \\
\text { ensayos, etc. }\end{array}$ & 0,321 & 0,337 \\
\hline 83 & Servicios sociales de mercado & 0,380 & 0,247 \\
\hline 87 & Otras actividades recreativas, culturales y deportivas & 0,553 & 0,086 \\
\hline 88 & Actividades diversas de servicios personales & 0,337 & 0,035 \\
\hline & Promedio de las ramas de actividad & $\mathbf{0 , 2 9 5}$ & $\mathbf{0 , 3 9 7}$ \\
\hline
\end{tabular}

Fuente: Elaboración propia a partir datos extraídos del Sistema de Cuentas Económicas de Andalucía. Marco Input-Output 1995 (IEA; 1999). 
Dentro del grupo III se encuadran 22 ramas de actividad caracterizadas por producir inputs aplicados a otros procesos productivos y por carecer de encadenamientos hacia atrás significativos. Entre ellas se sitúan las siguientes actividades:

\section{CUADRO 4. \\ RAMAS PRODUCTIVAS TIPO III. \\ (Encadenamientos directos hacia atrás inferiores a la media y hacia adelante superiores a la media)}

\begin{tabular}{|c|l|c|c|}
\hline Código & \multicolumn{1}{|c|}{ Denominación } & $\begin{array}{c}\text { Encadenam. } \\
\text { hacia atrás }\end{array}$ & $\begin{array}{c}\text { Encadenam. } \\
\text { hacia adelante }\end{array}$ \\
\hline 2 & Cultivos de vid y olivo & 0,136 & 0,903 \\
\hline 3 & Otros cultivos y servicios agrarios & 0,198 & 0,627 \\
\hline 5 & Silvicultura y servicios relacionados & 0,195 & 0,634 \\
\hline 21 & Industria textil & 0,219 & 0,421 \\
\hline 25 & Industria del papel & 0,239 & 0,425 \\
\hline 27 & Refino de petróleo & 0,193 & 0,445 \\
\hline 30 & $\begin{array}{l}\text { Industria de la transformación del caucho y materias } \\
\text { plásticas }\end{array}$ & 0,210 & 0,617 \\
\hline 32 & Fabricación de productos cerámicos, azulejos, ladrillos & 0,293 & 0,644 \\
\hline 35 & yatras tierras cocidas para la construcción & 0,213 & 0,430 \\
\hline 36 & Construcción de maquinaria y equipo mecánico & 0,219 & 0,471 \\
\hline 48 & Producción y distribución de gas y vapor de agua & 0,112 & 0,878 \\
\hline 51 & Preparación, instalación y acabado de obras & 0,280 & 0,437 \\
\hline 61 & Transporte aéreo & 0,290 & 0,863 \\
\hline 63 & Correos y telecomunicaciones & 0,112 & 0,535 \\
\hline 64 & Intermediación financiera & 0,174 & 0,685 \\
\hline 68 & Alquiler de maquinaria, equipos y otros efectos & 0,202 & 0,899 \\
\hline 69 & Actividades informáticas & 0,064 & 0,800 \\
\hline 70 & Investigación y desarrollo & 0,081 & 0,966 \\
\hline 73 & Publicidad & 0,074 & 0,952 \\
\hline 74 & Servicios de investigación y seguridad & 0,090 & 0,930 \\
\hline 75 & Actividades industriales de limpieza & 0,214 & 0,919 \\
\hline 76 & Otros servicios a las empresas & 0,136 & 0,903 \\
\hline & Promedio de las ramas de actividad & $\mathbf{0 , 2 9 5}$ & $\mathbf{0 , 3 9 7}$ \\
\hline
\end{tabular}

Fuente: Elaboración propia a partir de datos extraídos del Sistema de Cuentas Económicas de Andalucía. Marco Input-Output 1995 (IEA; 1999). 
Tres ramas agrarias: el cultivo de la vid y el olivo, otros cultivos agrarios y servicios agrarios y la silvicultura.

Un grupo heterogéneo de ramas industriales, entre ellas dos energéticas (refino de petróleo y la producción y distribución de gas y vapor de agua) y algunas actividades relacionadas con la construcción (como la fabricación de productos metálicos, la fabricación de productos cerámicos, azulejos, ladrillos y otras tierras cocidas para la construcción o la preparación, instalación y acabado de obras).

Algunos servicios, principalmente los orientados a las empresas, como los servicios financieros, los informáticos, las telecomunicaciones, las actividades de investigación y desarrollo, las actividades industriales de limpieza o la publicidad.

Por último, en el grupo IV se sitúan las ramas productivas con una reducida capacidad de arrastre y que producen un output destinado esencialmente a la demanda final. Se trata, por lo tanto, de ramas de actividad que carecen de importancia estratégica dentro del sistema productivo regional, en la medida en que presentan débiles conexiones con el resto del aparato productivo. Es éste el más nutrido de los grupos (alberga un total de 31 ramas) y en él figuran la mayor parte de las industrias de nivel tecnológico alto y medio-alto. Es el caso de la fabricación de otros productos químicos, la fabricación de máquinas de oficina y equipos informáticos, la fabricación de material eléctrico, la fabricación de material electrónico y aparatos de radio y televisión, la fabricación de equipos médicos y material de precisión o óptica, la fabricación de vehículos de motor, remolques y semirremolques y la fabricación de otro material de transporte.

En algunos casos las empresas de estos sectores actúan como enclaves cuyos inputs proceden del exterior y cuyo output tiene como destino principal la exportación. Es éste el caso de la fabricación de material electrónico y equipos de radio y televisión, la fabricación de equipo médico y aparatos de precisión, óptica, etc., la metalurgia o la fabricación de vehículos de motor, remolques y semirremolques. Esta falta de integración en el sistema productivo regional explicaría que, frente a lo que se comprueba en otras Comunidades Autónomas, las empresas de alta intensidad tecnológica instaladas en Andalucía no estén generando aparentemente externalidades positivas que impulsen el incremento de la productividad en otras ramas industriales como resultado de efectos de derrame tecnológico ${ }^{8}$.

8. Casillas Bueno, J. C. y Galán González, J. L. (1999): "Evolución y cambio de la industria andaluza en las dos últimas décadas”, Boletín Económico de Andalucía, n 26, Consejería de Economía y Hacienda, p. 213. 


\section{CUADRO 5. \\ RAMAS PRODUCTIVAS TIPO IV.}

(Encadenamientos directos hacia atrás y hacia adelante inferiores a la media)

\begin{tabular}{|c|c|c|c|}
\hline Código & Denominación & $\begin{array}{l}\text { Encadenam. } \\
\text { hacia atrás }\end{array}$ & $\begin{array}{c}\text { Encadenam. } \\
\text { hacia adelante }\end{array}$ \\
\hline 1 & Cultivos de hortalizas y frutas & 0,234 & 0,060 \\
\hline 4 & Producción ganadera y caza & 0,265 & 0,321 \\
\hline 6 & Pesca & 0,274 & 0,269 \\
\hline 17 & Industrias de otros productos alimenticios & 0,285 & 0,227 \\
\hline 20 & Industria del tabaco & 0,120 & 0,001 \\
\hline 22 & Industria de la confección y de la peletería & 0,253 & 0,133 \\
\hline 23 & Industria del cuero y del calzado & 0,275 & 0,203 \\
\hline 29 & Fabricación de otros productos químicos & 0,216 & 0,127 \\
\hline 34 & Metalurgia & 0,155 & 0,172 \\
\hline 37 & $\begin{array}{l}\text { Fabricación de máquinas de oficina y equipos } \\
\text { informáticos }\end{array}$ & 0,290 & 0,003 \\
\hline 38 & Fabricación de maquinaria y material eléctrico & 0,123 & 0,322 \\
\hline 39 & $\begin{array}{l}\text { Fabricación de material electrónico y equipos de } \\
\text { radio y televisión }\end{array}$ & 0,150 & 0,236 \\
\hline 40 & $\begin{array}{l}\text { Fabricación de equipo médico y aparatos de precisión, } \\
\text { óptica, etc. }\end{array}$ & 0,125 & 0,196 \\
\hline 41 & $\begin{array}{l}\text { Fabricación de vehículos de motor, remolques y } \\
\text { semirremolques }\end{array}$ & 0,185 & 0,068 \\
\hline 43 & Fabricación de otro material de transporte & 0,180 & 0,032 \\
\hline 45 & Otras industrias manufactureras & 0,290 & 0,149 \\
\hline 52 & Comercio de vehículos y carburantes & 0,234 & 0,127 \\
\hline 53 & Reparación de vehículos de motor & 0,181 & 0,336 \\
\hline 54 & Comercio al por mayor e intermediarios & 0,178 & 0,310 \\
\hline 55 & $\begin{array}{l}\text { Comercio al por menor y reparación de efectos } \\
\text { personales y domésticos }\end{array}$ & 0,294 & 0,042 \\
\hline 56 & Hoteles, pensiones y otros tipos de hospedaje & 0,254 & 0,156 \\
\hline 58 & Transporte por ferrocarril & 0,190 & 0,091 \\
\hline 67 & Actividades inmobiliarias & 0,065 & 0,253 \\
\hline
\end{tabular}




\begin{tabular}{|c|l|c|c|}
\hline Código & \multicolumn{1}{|c|}{ Denominación } & $\begin{array}{c}\text { Encadenam. } \\
\text { hacia atrás }\end{array}$ & $\begin{array}{c}\text { Encadenam. } \\
\text { hacia adelante }\end{array}$ \\
\hline 77 & $\begin{array}{l}\text { Administración pública, defensa y seguridad social } \\
\text { obligatoria }\end{array}$ & 0,233 & 0,000 \\
\hline 78 & Educación no de mercado & 0,037 & 0,000 \\
\hline 79 & Educación de mercado & 0,233 & 0,068 \\
\hline 80 & Actividades sanitarias y veterinarias no de mercado & 0,112 & 0,000 \\
\hline 81 & Actividades sanitarias y veterinarias de mercado & 0,159 & 0,100 \\
\hline 82 & Servicios sociales no de mercado & 0,137 & 0,000 \\
\hline 84 & Actividades de saneamiento público & 0,275 & 0,237 \\
\hline 89 & Hogares que emplean personal doméstico & 0,000 & 0,000 \\
\hline & Promedio de las ramas de actividad & 0,295 & 0,397 \\
\hline
\end{tabular}

Fuente: Elaboración propia a partir de datos extraídos del Sistema de Cuentas Económicas de Andalucía. Marco Input-Output 1995 (IEA; 1999).

En conclusión, por su importancia cuantitativa y su grado de integración en la estructura productiva, puede afirmarse que el sistema productivo andaluz se articula básicamente en torno a tres grandes complejos productivos: el agroalimentario, la construcción y los servicios turísticos, jugando también un papel relevante las ramas energéticas y los servicios a las empresas.

\section{LOS COMPLEJOS AGROALIMENTARIO, DE LA CONSTRUCCIÓN Y TURÍSTICO}

Los sectores productivos que presentan interrelaciones fuertes pueden conformar complejos productivos diferenciados en el conjunto del sistema productivo de la unidad socioeconómica de referencia, sea una región o una nación. Para identificar estas cadenas de producción se puede recurrir a los coeficientes de Streit, que proporcionan una estimación de la intensidad de las relaciones intersectoriales, distinguiendo las ligazones específicas de oferta y demanda.

Las ligazones específicas de demanda (LED) entre dos ramas se calculan como cociente entre la parte de la producción que el sector $i$ entrega al sector $j$ y el conjunto de todos los consumos intermedios del sector $j$. 


$$
L E D_{i j}=\frac{x_{i j}}{\sum_{i=1}^{n} x_{i j}}
$$

Por otro lado, las ligazones específicas de oferta (LEO) entre dos ramas se calculan mediante el cociente entre el consumo intermedio que el sector $j$ hace de productos procedentes del sector $i$ y el conjunto de los outputs intermedios del sector $i$.

$$
L E O_{i j}=\frac{x_{i j}}{\sum_{j=1}^{n} x_{i j}}
$$

Estos dos tipos de ligazones proyectados sobre la relación entre dos sectores cualesquiera dan lugar a cuatro posibles conexiones: $L E D_{i j}, L E D_{j i}, L E O_{i j}, L E O_{j i}$. El coeficiente de ligazones específicas de Streit es simplemente la media aritmética de esas cuatro ligazones específicas, como sigue:

$$
C E S_{i j}=\frac{1}{4}\left(L E O_{i j}+L E O_{j i}+L E D_{i j}+L E D_{j i}\right)
$$

donde necesariamente: $C E S_{i j}=C E S_{j i}$

Para poder discriminar cuáles son las relaciones intersectoriales más importantes puede establecerse un umbral mínimo para cada rama, de modo que un sector $j$ se considera incluido en la cadena productiva de un sector $i$ siempre que el $C E S_{i j}$ es superior al umbral establecido.

Conforme al procedimiento expuesto, se han calculado las ligazones específicas de oferta y demanda entre todos los sectores y, a continuación, los coeficientes de ligazones específicas de Streit. A partir de la observación de los valores de estos coeficientes para cada rama productiva se han identificado aquéllas que forman parte de su cadena de producción, adoptando como criterio el considerar incluido en la cadena productiva de un sector a todas aquellas ramas vinculadas a él por un coeficiente de ligazones específicas de Streit superior o igual a 0,15.

De esta forma, se pueden señalar con mayor exactitud las ramas que conforman los tres grandes complejos productivos sobre los que se sostiene el sistema productivo andaluz: el complejo agroalimentario, el complejo de la construcción y el complejo turístico. 


\subsection{El complejo agroalimentario}

Está constituido esencialmente por 14 ramas productivas que suponen el 13\% del PIB andaluz y el $43 \%$ de las exportaciones y pueden clasificarse en dos grupos:

a) El sector agrario y la pesca, compuesto por las siguientes seis ramas: los cultivos de hortalizas y frutas, los cultivos de la vid y el olivo, otros cultivos y servicios agrarios, la ganadería y la caza, la silvicultura y los servicios relacionados y la pesca.

b) Las industrias alimentarias y de bebidas, comprendiendo la industria cárnica; la elaboración de conservas de pescado y vegetales; la fabricación de grasas y aceites; las industrias lácteas; las industrias de molinería, pan, galletas y pastelería; las industrias de otros productos alimenticios; la elaboración de vinos y alcoholes y la elaboración de cerveza y bebidas no alcohólicas.

\section{CUADRO 6.}

IMPORTANCIA CUANTITATIVA DEL COMPLEJO AGROALIMENTARIOENLAECONOMÍA ANDALUZA. PORCENTAJES RESPECTO AL VAB Y A LAS EXPORTACIONES TOTALES.

\begin{tabular}{|c|l|c|c|}
\hline Código & \multicolumn{1}{|c|}{ Denominación } & VAB & Export. \\
\hline 1 & Cultivos de hortalizas y frutas & 2,36 & 9,54 \\
\hline 2 & Cultivos de vid y olivo & 2,83 & 0,73 \\
\hline 3 & Otros cultivos y servicios agrarios & 1,12 & 1,82 \\
\hline 4 & Producción ganadera y caza & 1,22 & 2,65 \\
\hline 5 & Silvicultura y servicios relacionados & 0,18 & 0,28 \\
\hline 6 & Pesca $\quad$ Total Sector Agrario y Pesca & 0,33 & 0,21 \\
\hline & & $\mathbf{8 , 0}$ & $\mathbf{1 5 , 2}$ \\
\hline 12 & Industria cárnica & 0,54 & 1,15 \\
\hline 13 & Elaboración de conservas de pescado y de vegetales & 0,45 & 2,84 \\
\hline 14 & Fabricación de grasas y aceites & 0,68 & 11,26 \\
\hline 15 & Industrias lácteas & 0,29 & 1,01 \\
\hline 16 & Industrias de molinería, pan, galletas y pastelería & 0,76 & 2,66 \\
\hline 17 & Industrias de otros productos alimenticios & 0,79 & 4,59 \\
\hline 18 & Elaboración de vinos y alcoholes & 0,51 & 3,49 \\
\hline 19 & Elaboración de cerveza y bebidas no alcohólicas & 0,48 & 1,21 \\
\hline & Total industrias alimentarias & $\mathbf{4 , 5}$ & $\mathbf{2 8 , 2}$ \\
\hline & Total Complejo Agroalimentario & $\mathbf{1 2 , 5}$ & $\mathbf{4 3 , 4}$ \\
\hline
\end{tabular}

Fuente: Elaboración propia a partir de datos extraídos del Sistema de Cuentas Económicas de Andalucía. Marco Input-Output 1995 (IEA; 1999). 
Articulación intersectorial y complejos productivos. Un análisis del sistema productivo... 25

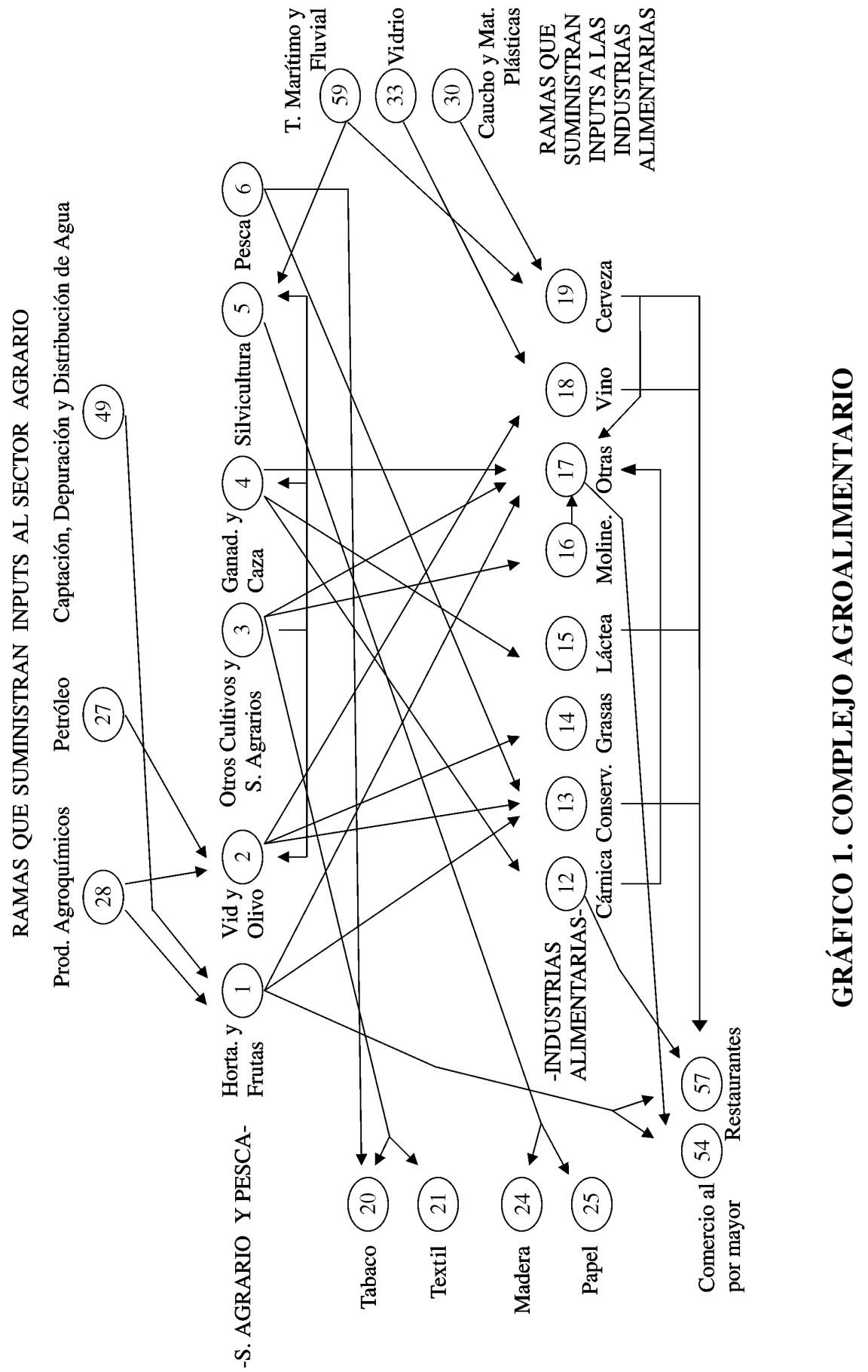


Estas ramas presentan vínculos importantes con otros sectores que las proveen de suministros básicos necesarios para su actividad o les prestan determinados servicios intermedios. En concreto, las ramas agrarias, en general, demandan inputs procedentes del refino de petróleo, la fabricación de productos agroquímicos (fertilizantes y fitosanitarios) y la captación, depuración y distribución de agua. Por su parte, entre las industrias alimentarias, la industria de la cerveza y bebidas no alcohólicas realiza compras abundantes a la industria de la transformación del caucho y materias plásticas y la industria del vino y de alcoholes depende para el embotellado de sus productos de la industria del vidrio. Igualmente, la silvicultura y la industria de la cerveza y bebidas no alcohólicas demandan servicios de transporte marítimo y fluvial.

Por otro lado, también existen vínculos importantes entre algunas ramas del complejo agroalimentario y otras actividades industriales que emplean inputs procedentes del sector primario. Es el caso de los encadenamientos entre la industria textil y la rama otros cultivos agrarios, por las compras de fibras textiles de origen vegetal, los de la industria del tabaco con el cultivo de tabaco o la pesca, o los de la industria de la madera y del papel con la silvicultura. Finalmente, es interesante señalar la relación que mantienen el complejo agroalimentario y el complejo turístico a través de los suministros de materias primas con destino a restaurantes, bares y otros establecimientos similares.

\subsection{El complejo de la construcción}

Se conforma en torno a dos ramas productivas: principalmente, la construcción de inmuebles y obras de ingeniería civil y, en segundo lugar, la preparación, instalación y acabado de obras que, conjuntamente, representan el 8,9\% del PIB regional. A su vez, forman parte de este complejo industrial una serie de ramas proveedoras de inputs necesarios para la actividad de la construcción, como la extracción de minerales no metálicos, ni energéticos; las industrias de la madera y del corcho (excepto muebles); la fabricación de otros productos químicos; la fabricación de cemento, cal, yeso y sus derivados; la fabricación de productos cerámicos, azulejos, ladrillos y otras tierras cocidas para la construcción; las industrias del vidrio y de la piedra; la fabricación de productos metálicos o la fabricación de maquinaria y material eléctrico. Estas ramas mantienen un estrecho grado de integración con el sector de la construcción, como prueba el creciente uso de componentes prefabricados que se entregan a pie de obra, lo que implica la transferencia de parte del valor añadido a las propias industrias proveedoras?.

9. Rodríguez, José Javier (1995): "El Complejo de la Construcción en Andalucía (Una aproximación desde la TIOAN-90", en Contabilidad Regional y Tablas Input-Output de Andalucía 1990. Análisis de resultados. Volumen 2, IEA, Sevilla, p. 456. 


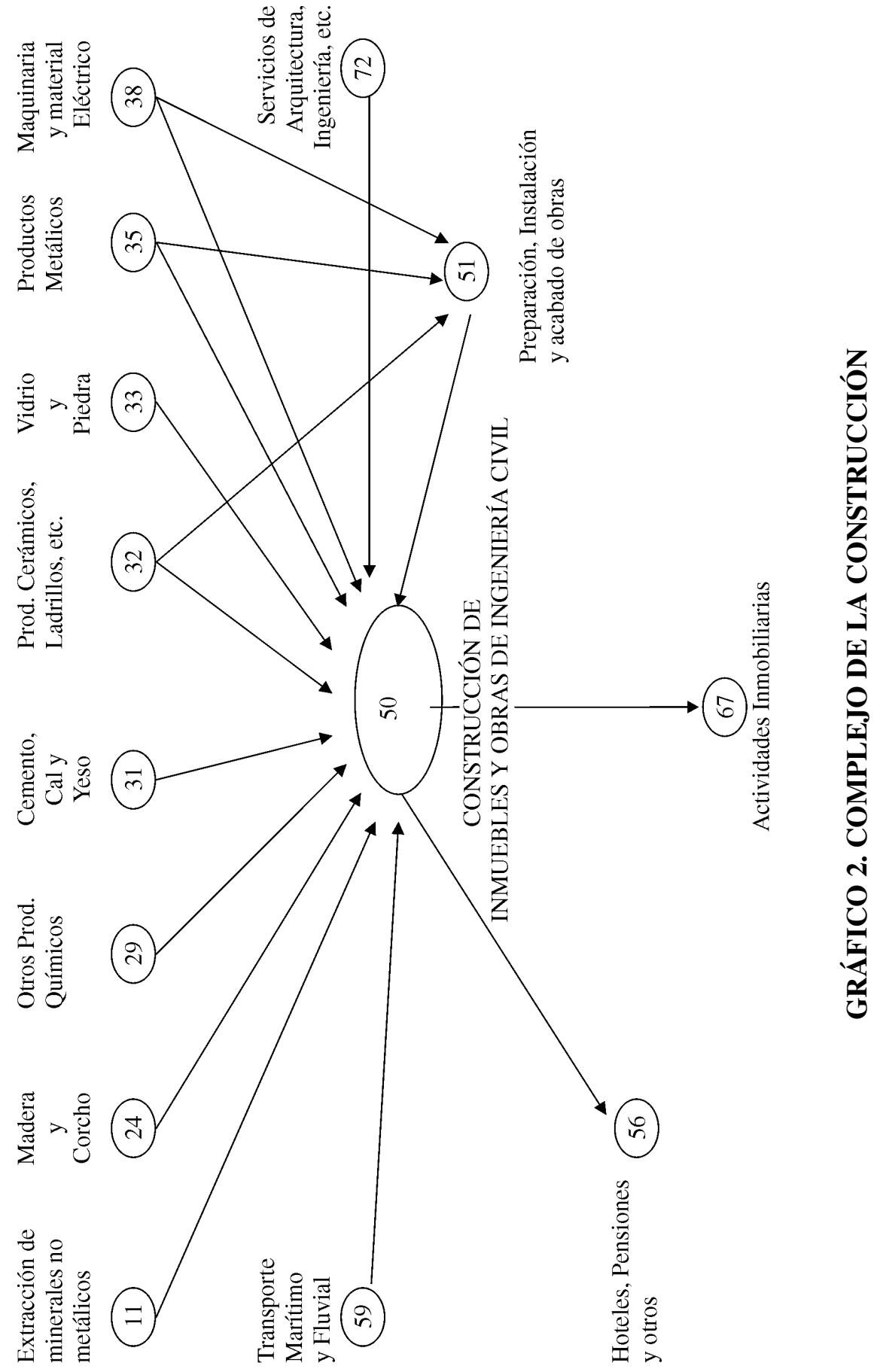


El sector de la construcción también recurre en su proceso productivo a dos ramas de servicios: el transporte marítimo y fluvial y los servicios técnicos de arquitectura, ingeniería, ensayos, etc. Por el contrario, los vínculos intersectoriales del complejo de la construcción por el lado de la oferta no son muy importantes, ya que el principal destino de su actividad es la demanda final, en concreto, la formación bruta de capital. No obstante, destaca, en este sentido, el papel desempeñado por dos ramas del complejo turístico: las actividades inmobiliarias y los servicios de hoteles, pensiones y otros.

\subsection{El complejo turístico}

La delimitación de las ramas productivas que podrían considerarse dentro del sector turismo resulta un problema, puesto que no existe una sola actividad que por su propia naturaleza derive en un servicio de carácter turístico ${ }^{10}$. Por ejemplo, el gasto en restaurantes, y otros establecimientos para beber y comer realizado por los turistas no puede separarse del realizado por los residentes o por los trabajadores que, por motivos de trabajo, tienen que desplazarse fuera de su lugar de residencia. No obstante, es posible identificar una serie de actividades que eventualmente tienen un carácter turístico.

Hoteles, pensiones y otros tipos de hospedaje.

Restaurantes y otros lugares donde comer y beber.

Las actividades de transporte, en lo que se refiere al transporte de viajeros.

Otras actividades anexas a los transportes, donde se incluyen, por ejemplo, la explotación de aparcamientos y los servicios de las agencias de viajes.

Actividades inmobiliarias, en concreto, las relacionadas con el alquiler de apartamentos de temporada.

El alquiler de maquinaria, de equipos y otros efectos, aunque sólo en lo que respecta al alquiler de automóviles.

Otras actividades recreativas, culturales y deportivas.

En menor medida, también tienen en ocasiones un carácter turístico el comercio al por menor, las reparaciones de efectos personales y domésticos y las actividades diversas de servicios personales.

En conjunto, todas estas actividades supondrían un 18,2\% del PIB andaluz (o un $27,6 \%$ si se incluyen el comercio al por menor, las reparaciones y las actividades diversas de servicios personales). Lógicamente, estas cifras estarían infladas por el hecho de considerar como turísticas actividades que, pese a estar recogidas en esas ramas productivas, no tienen ese carácter.

10. Rodríguez, Jesús y Villegas, Pedro Pablo (1995): "La economía turística andaluza a la luz de las tablas inputoutput de Andalucía 1990"; en Contabilidad Regional y Tablas Input-Output de Andalucía 1990. Análisis de resultados. Volumen 2, IEA, Sevilla, p. 504. 
Articulación intersectorial y complejos productivos. Un análisis del sistema productivo... 29

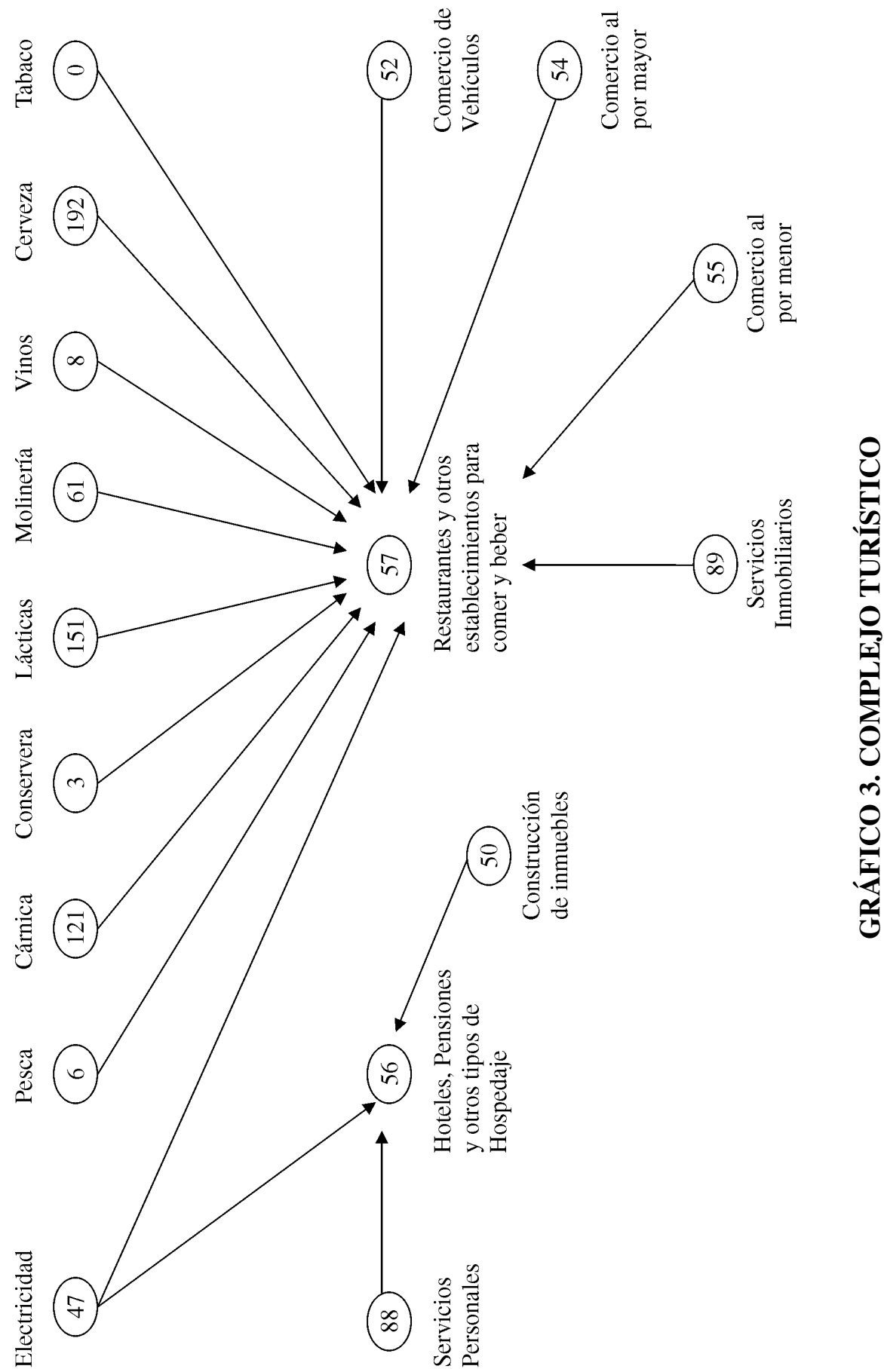


En el gráfico anterior puede apreciarse la existencia de vínculos estrechos entre el complejo turístico, considerando la hostelería ${ }^{11}$ como su núcleo central, con otras ramas de actividad que le proveen de inputs intermedios, como la pesca, las industrias alimentarias (la industria cárnica, las industrias de elaboración de conservas, las industrias lácteas, la industria de la molinería, el pan, etc., la industria de elaboración de vinos y otras bebidas alcohólicas, la industria de la cerveza y bebidas no alcohólicas), la industria del tabaco, la producción y distribución de electricidad, las actividades diversas de servicios personales, el comercio de vehículos, el comercio al por mayor e intermediarios, el comercio al por menor y las reparaciones o las actividades inmobiliarias. De este modo, se comprueba la conexión existente entre la actividad turística y los complejos agroalimentario y de la construcción.

\section{CONCLUSIONES}

$\mathrm{Al}$ analizar el sistema productivo andaluz se revela como uno de sus rasgos fundamentales un dualismo definido por la coexistencia de dos modos de producción: Por un lado, un modo de producción tradicional y endógeno, conformado por un núcleo de actividades muy integradas en el sistema productivo regional, entre las que destacan los complejos productivos agroalimentario, de la construcción y turístico. En el transcurso de este trabajo se ha profundizado en el análisis de la estructura interna de estos complejos y de las interrelaciones que mantienen entre ellos. Por otro lado, nos encontramos con un sector industrial moderno e intensivo en capital, liderado por iniciativas empresariales foráneas -nacionales o extranjeras-, que en muchos casos se localiza en la región para aprovechar ventajas de costes laborales y se encuentra frecuentemente desvinculado del resto del sistema productivo andaluz (metalurgia, material de transporte, maquinaria y material eléctrico y electrónico, etc.).

La debilidad del sector industrial y el dualismo en la estructura productiva regional determinan la desarticulación que con carácter general caracteriza al sistema productivo andaluz. La desvertebración productiva de la economía andaluza bloquea la difusión de los procesos de crecimiento y determina el desaprovechamiento de las economías externas estáticas y dinámicas implícitas en los encadenamientos productivos potenciales y no explotados.

En la actualidad, el proceso de globalización está impulsando no sólo la competencia entre empresas, sino también entre territorios que tratan de conseguir una posición favorable en la división internacional del trabajo a escala global. Para ello, las regiones procuran fortalecer sus ventajas comparativas y cimentar sobre ellas pautas de especialización que las integren en la economía global en condiciones

11. Se considera aquí la hostelería en sentido amplio, incluyendo la oferta de alojamiento así como la actividad de restaurantes, bares y establecimientos similares. 
ventajosas. En este contexto, la economía andaluza necesita profundos cambios estructurales sin los cuales el mero crecimiento no hará sino acentuar la desvertebración de la economía regional. En este sentido, resulta estratégica la integración en el sistema productivo andaluz de los enclaves industriales en actividades de alto nivel tecnológico. Para que estas empresas y estos sectores aporten más al desarrollo andaluz deben articularse con el tejido productivo autóctono, para aprovechar así las externalidades, generar efectos de arrastre y facilitar el derrame tecnológico a través de la difusión de las innovaciones. Así mismo, se deben reforzar o restablecer las ventajas comparativas en sectores tradicionales de carácter endógeno, como el complejo agroalimentario o las actividades turísticas a través de la diferenciación del producto, la calidad, el diseño, el marketing, la comercialización o la tecnología. La economía andaluza no puede desaprovechar el potencial de estas actividades, sino que debe dinamizarlas y cimentar sobre ellas un proceso de desarrollo endógeno basado en el saber hacer, en los conocimientos acumulados, en la cualificación de los recursos humanos, en la tradición empresarial y en la imbricación de estas actividades en el sistema productivo regional.

\section{BIBLIOGRAFÍA}

AURIOLES MARTÍN, J. (1989): Claves actuales de la Economía Andaluza, "Cuadernos Andaluces”. Editorial Librería Ágora. Málaga.

DELGADO CABEZA, M. (1981): Dependencia y marginación de la economía andaluza. Monte de Piedad y Caja de Ahorros de Córdoba. Córdoba.

DELGADOCABEZA, M. (1995): "Las relaciones con el exterior de la estructura productiva andaluza. Análisis de las necesidades de importación. 1980-1990"; en Contabilidad Regional y Tablas Input-Output de Andalucía 1990. Análisis de resultados. IEA. Sevilla. pp. 273-331.

CASILLAS BUENO, C. Y GALÁN GONZÁLEZ, J. L. (1999): "Evolución y cambio de la industria andaluza en las dos últimas décadas". Boletín Económico de Andalucía. $\mathrm{N}^{\circ}$ 26. Consejería de Economía y Hacienda. pp. 203- 218.

GARCÍA RECHE, A. (1998): "Especialización productiva, dinámica sectorial e innovación: Otra perspectiva para el empleo en España”. Economía Industrial. $\mathrm{n}^{\circ} 324$.

GLAESER, EDWARD L.; KALLAL, HEDI D.; SCHEINKMAN, JOSÉ A y SHLEIFER, ANDREI (1992): "Growth in Cities". Journal of Political Economy. vol. 100. nº 6. pp. 1126-1152.

GUZMÁN CUEVAS, J. y LIÑÁN-ALCALDE, F. (1997): "Qualitative analysis of the entrepreneur. An application to southern Spain", comunicación presentada a European 
Urban and Regional Research Network Conference, de la Regional Studies Association. Franckfurt del Oder (Alemania). 20-23 septiembre 1997.

GUZMÁN CUEVAS, J.; LIÑÁN ALCALDE, F.; ROMERO LUNA, I. y SANTOS CUMPLIDO, J. (2000): "Las PYMES y la calidad del tejido productivo andaluz". Boletín Económico de Andalucía. №27-28, Consejería de Economía. Junta de Andalucía. pp. 43-56.

HIRSCHMAN, ALBERT O. (1961): La estrategia del desarrollo económico. Fondo de Cultura Económica. México D. F.

INSTITUTO DEESTADÍSTICA DE ANDALUCÍA (1999): Sistema de Cuentas Económicas de Andalucía. Marco Input-Output. 1995. IEA. Sevilla.

de LUCIO, JUAN J., JOSÉ A, HERCE y ANA GOICOLEA (1998): "The Effects of Externalities on Value Added and Productivity Growth in Spanish Industry", FEDEA, Documento de Trabajo 98-05.

MORAL PAJARES, E. (1998): Ajuste del Comercio Exterior Andaluz tras la Adhesión de España a la Unión Europea. Universidad de Jaén. Jaén.

OTERO MORENO, J. M. (1995): "Multiplicadores de la economía andaluza: conceptos, medida y guía de aplicación", en Contabilidad Regional y Tablas Input-Output de Andalucía 1990. Análisis de resultados. IEA. Sevilla. pp. 145-273.

PULIDO, ANTONIO y FONTELA, EMILIO (1993): Análisis Input-Output. Modelos, datos y aplicaciones. Ed. Pirámide. Madrid.

RODRÍGUEZ, JESÚS Y VILLEGAS, PEDRO PABLO (1995): "La economía turística andaluza a la luz de las tablas input-output de Andalucía 1990”; en Contabilidad Regional y Tablas Input-Output de Andalucía 1990. Análisis de resultados. Volumen 2. IEA. Sevilla. pp. 499-533.

RODRÍGUEZ, JOSÉ JAVIER (1995): “El Complejo de la Construcción en Andalucía (Una aproximación desde la TIOAN-90", en Contabilidad Regional y Tablas Input-Output de Andalucía 1990. Análisis de resultados. Volumen 2. IEA. Sevilla. pp. 453-499. 